\title{
Late-Onset Primary Intestinal Lymphangiectasia Successfully Managed with Octreotide: A Case Report
}

\author{
Kotaro Suehiro, MD, Noriyasu Morikage, MD, Masanori Murakami, MD, Osamu Yamashita, MD, and \\ Kimikazu Hamano, MD
}

\begin{abstract}
We report a case of a patient with late-onset primary lymphangiectasia whose persistent diarrhoea was successfully managed with octreotide. A 63 year-old man visited our clinic with a complaint of worsening general edema. Gastrointestinal endoscopy revealed typical whitish jejunal villi, which suggested primary intestinal lymphangiectasia. Despite a diet, supplemented with medium-chain triglycerides; antiplasmin therapy; oral corticosteroids; and surgery, including pericardial window and lymphaticovenous anastomoses; his symptoms, including watery diarrhoea, showed no improvement. After administration of octreotide, his persistent diarrhoea resolved within a couple of days. Octreotide was continued for 2 months. Thereafter, his diarrhoea has not recurred for 6 months.
\end{abstract}

Keywords: intestinal lymphangiectasia, octreotide, protein-losing enteropathy

\section{INTRODUCTION}

$\mathrm{P}$ rimary intestinal lymphangiectasia (PIL), first described by Waldmann et al. in 1961, ${ }^{1)}$ is a rare disorder characterized by dilated intestinal lacteals that cause leakage of the lymph into the small bowel lumen, which results in protein-losing enteropathy (PLE). ${ }^{2)}$ Most patients are diagnosed with PIL in childhood. Clinical presentation of the disease includes general edema, diarrhea, and symptoms associated with PLE and malabsorption syndrome. We report a case of a patient who presented with PIL for the first time at the age of 60 years, and persistent diarrhea of the patient was dramatically improved with octreotide.

Department of Surgery and Clinical Science, Division of Vascular Surgery, Yamaguchi University Graduate School of Medicine, Ube, Yamaguchi, Japan

Received: November 7, 2011; Accepted: December 17, 2011 Corresponding author: Kotaro Suehiro, MD. Department of Surgery and Clinical Science, Division of Vascular Surgery, Yamaguchi University Graduate School of Medicine, 1-1-1, Minamikogushi, Ube, Yamaguchi 755-8505, Japan

Tel: +81-836-22-2260, Fax: +81-836-22-2423

E-mail: ksuehiro-circ@umin.ac.jp

\section{Case Report}

A 63 year old man was referred to our clinic because of progressive, general edema and a recent increase in body weight by $5 \mathrm{~kg}$. He reported swelling that he had noticed for the first time in the upper extremities, in the face within 3 years from the onset, and subsequently in the lower extremities. Physical examination revealed pitting edema of the arms and legs, as well as of the face. No remarkable edema was seen in the body trunk during his first visit. Consultations with physicians did not indicate particular problems in the cardiac, renal, and hepatic function and neither in thyroid function. He had surgery for bilateral hydrocele testis at 40 years of age; otherwise, his medical and family histories were unremarkable.

Laboratory tests revealed the following values (normal values in parentheses): serum total protein, $5.3 \mathrm{~g} / \mathrm{dL}$ $(6.8-8.3 \mathrm{~g} / \mathrm{dL})$ and albumin, $3.0 \mathrm{mg} / \mathrm{dL}(3.7-4.7 \mathrm{mg} / \mathrm{dL})$. Immunoglobulin $\mathrm{G}(\mathrm{IgG})$ concentration, $741.0 \mathrm{mg} / \mathrm{dL}$ (870-1700 mg/dL), depleted while maintaining IgA $151.0 \mathrm{mg} / \mathrm{dL}(110-410 \mathrm{mg} / \mathrm{dL})$ and $\operatorname{IgM} 39.9 \mathrm{mg} / \mathrm{dL}$ (33-190 $\mathrm{mg} / \mathrm{dL})$ in the normal range. Lymphocytopenia $22.7 \%(30.0-46.0 \%)$ and hypocalcemia $2.20 \mathrm{mmol} / \mathrm{L}$ (2.30-2.65 mmol/L) were also observed. Lymphangioscintigraphy (LAS) in the extremities showed the absence of lymph trunks and inguinal and axillary nodes, with 

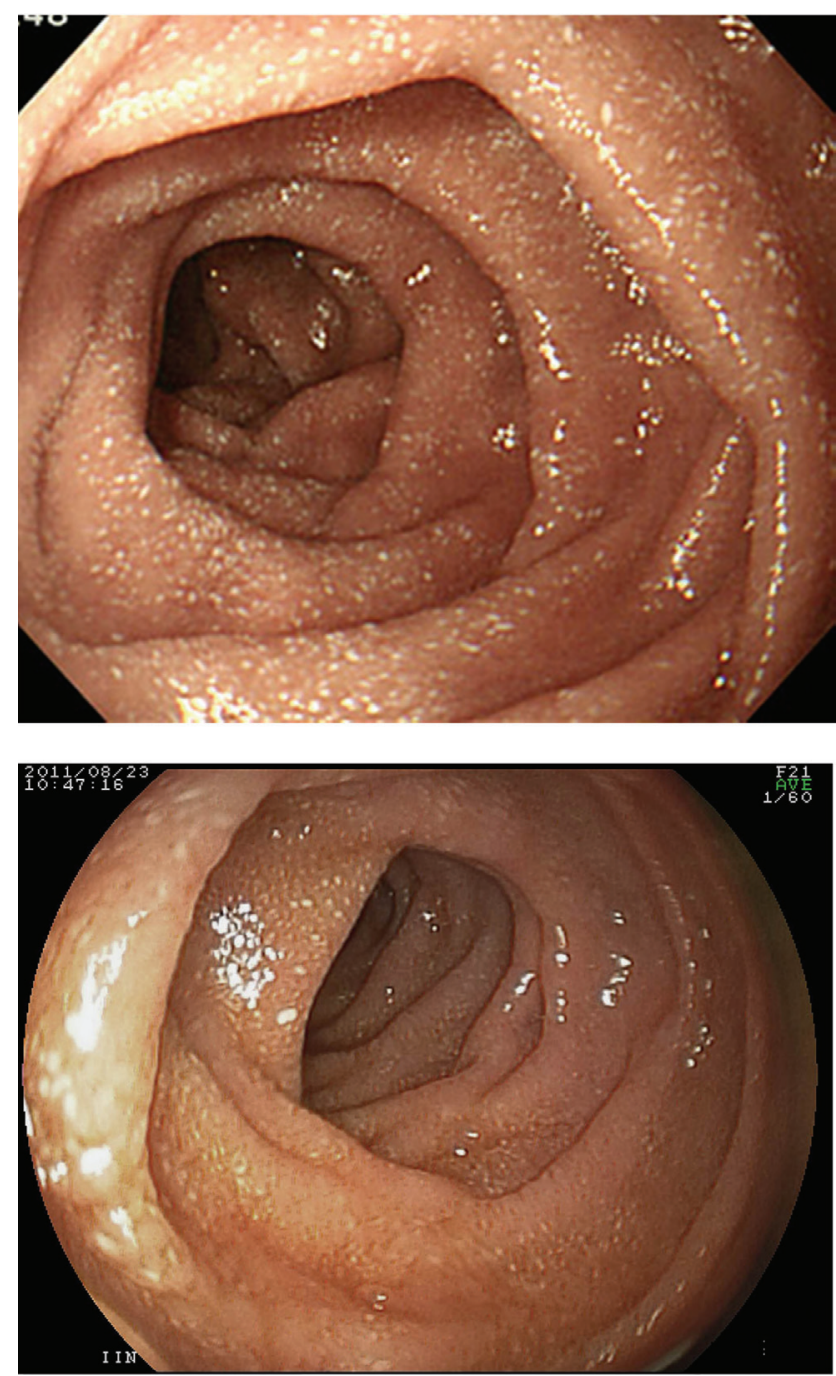

Fig. 1 Gastrointestinal endoscopy (Duodenum). Before treatment with octreotide, tiny scattered white spots, ie, whitish jejunal villi, indicating dilated lymphatics filled with fat droplets were seen on the entire duodenal wall. (A) Whitish jejunal villi markedly reduced even 6 months after discontinuation of octreotide. (B)

significant accumulation of contrast medium in distal extremities. Since his skin condition was preserved, it seemed that he had a complication of grade I primary lymphedema in the upper and the lower extremities.

Chest radiograph, computed tomography (CT) scan, and echocardiography showed pericardial and bilateral pleural effusion but few ascites. Magnetic resonance lymphography showed a patent thoracic duct and no dilatation of the cisterna chyli. No remarkable dilatation was found in the intraabdominal and retroperitoneal lymphatics. Gastrointestinal endoscopy and capsule endoscopy revealed typical whitish jejunal villi and intestinal edema (Fig. 1A). ${ }^{99 \mathrm{~m}} \mathrm{Tc}$-labeled human serum albumin ( $\left.{ }^{99 \mathrm{~m}} \mathrm{Tc}-\mathrm{HAS}\right)$ scintigraphy showed patchy leak of albumin in the duodenum and the entire small intestine (Fig. 2). Biopsies from the duodenal wall did not show a typical picture of dilated lymphatics in the lamina propria, ${ }^{3)}$ but instead showed mildly dilated lymphatics in the deeper layer. Since no other underlying diseases, including inflammatory bowel disease, infection, celiac disease, etc., were found in the gastrointestinal system of the patient, his condition was diagnosed as PLE due to PIL associated with primary lymphedema in the extremities.

A low-fat diet with supplementary medium-chain triglycerides (MCT), diuretics, and complex physical therapy for lymphedema were immediately initiated at the outpatient clinic. Although the serum albumin level had been maintained around $3.0 \mathrm{~g} / \mathrm{dL}$ or higher, the patient had iron-deficiency anemia and worsening lymphocytopenia and hypocalcemia. At around 6 months from his first visit, he complained of watery diarrhea 5 to 8 times/day, as well as increasing shortness of breath, which was caused by increasing pleural and pericardial effusion. His symptoms did not improve despite an increase in doses of diuretics and antiplasmin therapy (tranexamic acid $1 \mathrm{~g}, 3$ times/day) followed by the administration of oral corticosteroids (prednisolone, $5 \mathrm{mg} / \mathrm{day}$ ).

After a year of treatment at the outpatient clinic with no improvement in his condition, the patient was eventually admitted and kept on complete, total parenteral nutrition (TPN). Following cessation of oral intake, his watery diarrhea had stopped. We performed the videoassisted, thoracoscopic pericardial window procedure, for the reduction of right atrial pressure and microsurgical lymphaticovenous anastomoses in both groins, and we had expected that these procedures would have favorable effects on overall lymphatic drainage. Both pleural and pericardial effusions were serous and not chylous in nature. The inguinal lymphatics were dilated and filled with clear lymph, but not chyle. On postoperative day 5, namely after 12 days of TPN, light meal was re-started and then watery diarrhea recurred immediately. Finally, on post-operative day 8 , administration of $100 \mu \mathrm{g}$ of subcutaneous octreotide twice daily was initiated. From the next day, his diarrhea gradually disappeared, and eventually, he had normal bowel movements over a couple of days. Subsequently, octreotide was administered as a slow-release formulation (Sandostatin $\operatorname{LAR}^{\circledR} 20 \mathrm{mg}$ ), and he was discharged on postoperative day 17. Slow-release octreotide was injected once every 4 weeks at the outpatient clinic. After 3 injections, however, the patient was 

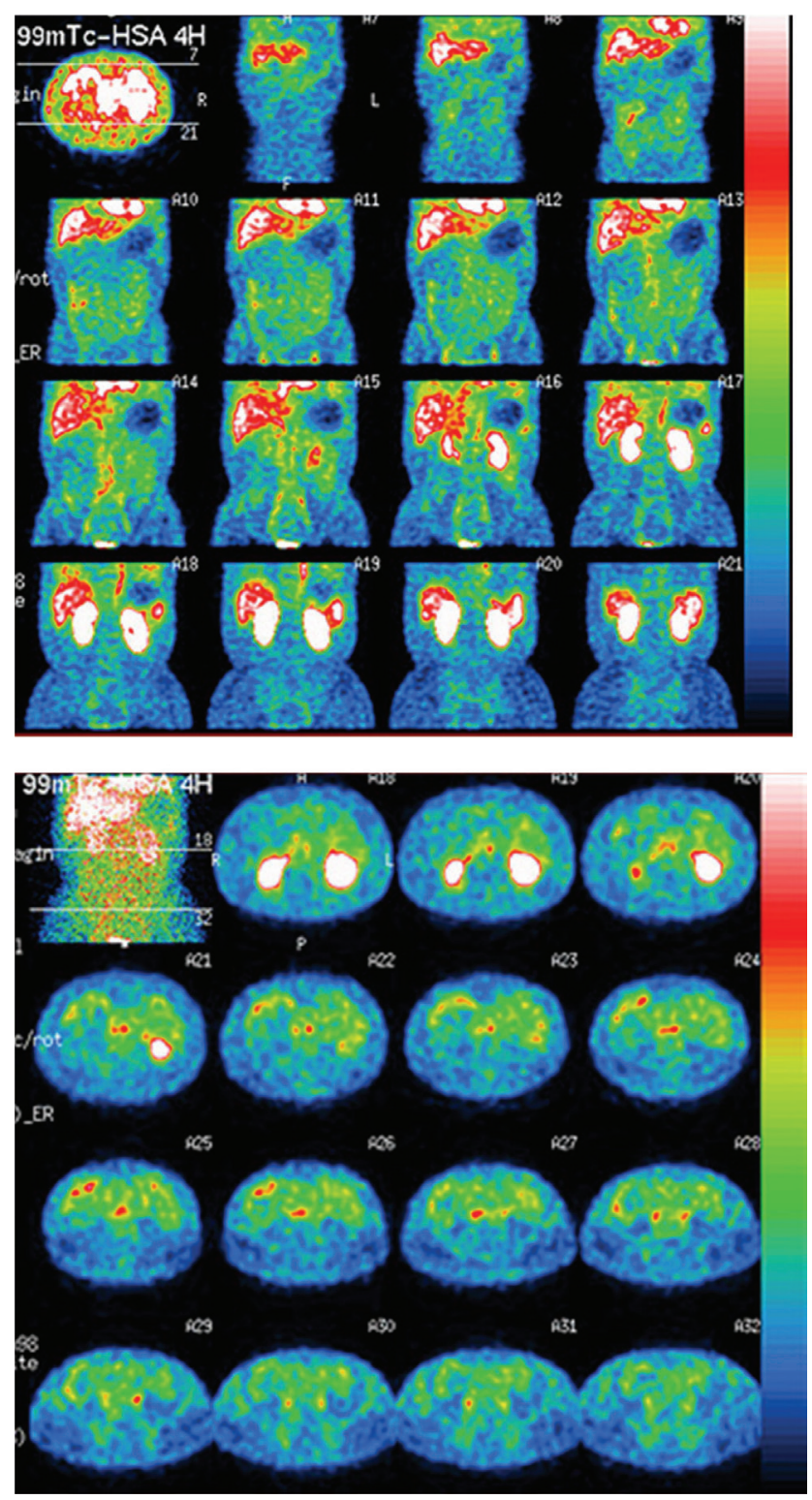

Fig. $2{ }^{99 m}$ Tc-labeled human serum albumin scintigraphy. Multiple and patchy leakage of albumin (seen as red spots) is scattered in the entire length of the duodenum and the small intestine.

unable to afford it, and the injection was discontinued.

At 8 months from discharge, 6 months after the discontinuation of slow-release octreotide, the patient has not experienced watery diarrhea, and recent gastrointestinal endoscopy showed reduced whitish jejunal villi in the duodenum (Fig. 1B), but mild hypoalbuminemia (3.3 g/dL) causing edema continues to persist.

\section{Discussion}

Since PIL can be asymptomatic, the exact prevalence of this disease, as well as its cause and pathogenesis, remain to be clarified. ${ }^{2,4)}$ PIL is usually diagnosed before 3 years of age, but may be diagnosed in adults. However, late presentation such as 60 years of age or older, similar to that in our patient, is very rare. Primary lymphedema in the extremities can be associated with PIL as in the case of our patient. The diarrhea in this patient started 6 months after his first visit. At the time of the first gastrointestinal endoscopy, however, intestinal edema had already been observed. Therefore, we speculate that inflammation and edema progressed and eventually, they caused watery diarrhea, which further worsened these symptoms.

Currently, treatment options for PIL are limited, and a definite treatment option remains to be established. ${ }^{2)}$ Diet supplemented with MCT is an essential component and should be continued throughout the life of the patient. Although antiplasmin therapy using tranexamic acid is effective in some patients, it was not effective in our patient. Higher doses of corticosteroids may be prescribed for PLE with underlying inflammatory or autoimmune diseases, but the efficacy of corticosteroids for PIL is uncertain. Since PIL can develop into uncontrollable intestinal bleeding or ileus which may require emergent small bowel resection in the later course of the disease, $\left.{ }^{5}\right)$ treatment with high-dose steroid was reserved as a last resort in our patient. Although small bowel resection may be effective, it is indicated only in cases of patients with localized PIL, and thus, it was not performed in our patient. Indication of lymphaticovenous anastomoses for this patient may be controversial. Although it was not described, there was one episode of cellulitis before admission, possibly due to lymphatic congestion. He had not experienced cellulitis since surgery; however, this might have been the result of an overall improvement in lymphatic drainage by the reduction of right atrial pressure in the pericardial window procedure.

Octreotide, a somatostatin analogue, has been shown to alleviate various types of diarrhea. Although little is known about the mechanism of action of somatostatin, particularly in the treatment of PIL, inhibition of gastrointestinal vasoactive peptides, stimulation of the autonomic nervous system ${ }^{6}$ ) and reduction in intestinal absorption of fats are the proposed mechanisms of action. ${ }^{7,8)}$ Sari et al. studied the long-term safety of octreotide in 6 patients for up to 37 months. ${ }^{9)}$ One patient had pancreatitis, but no other adverse effects were observed in the other patients.

The cost of continuing octreotide, which is not covered by insurance, is a serious problem in our country. Since hypoalbuminemia and general edema in our patient did 
not show sufficient improvement, we proposed to increase the doses of octreotide. However, the patient declined treatment for financial reasons. Sandostatin $\operatorname{LAR}^{\circledR}$ $20 \mathrm{mg}$ costs $\backslash 237000$, which is certainly not affordable for ordinary people every 4 weeks. Our results show that octreotide can exhibit favorable effects for up to 6 months along with pathological improvement. Kuroiwa et al. reported that discontinuing octreotide resulted in recurrence of symptoms, which could be improved by re-starting octreotide. ${ }^{10)}$ Therefore, although the regular administration is desirable, the short-term use of octreotide may be considered depending on the symptoms.

\section{Conclusion}

We reported the case of a patient with PIL causing PLE successfully treated with octreotide. Although it is not curative, octreotide seemed particularly useful in controlling diarrhea in our patient.

\section{ACKnowledgements}

We thank Dr Hideo Amano for generously providing follow-up pictures of gastrointestinal endoscopy.

\section{REFERENCES}

1) Waldmann TA, Steinfeld JL, Dutcher TF, et al. The role of the gastrointestinal system in "idiopathic hypoproteinemia”. Gastroenterology. 1961; 41: 197-207.
[Medline]

2) Vignes S, Bellanger J. Primary intestinal lymphangiectasia (Waldmann's disease). Orphanet J Rare Dis. 2008; 3: 5. [Medline] [CrossRef]

3) Asakura H, Miura S, Morishita T, et al. Endoscopic and histopathological study on primary and secondary intestinal lymphangiectasia. Dig Dis Sci 1981; 26: 312-20. [Medline] [CrossRef]

4) Freeman HJ, Nimmo M. Intestinal lymphangiectasia in adults. World J Gastrointest Oncol 2011; 3: 19-23. [Medline] [CrossRef]

5) Zhu LH, Cai XJ, Mou YP, et al. Partial enterectomy: treatment for primary intestinal lymphangiectasia in four cases. Chin Med J (Engl) 2010; 123: 760-4. [Medline]

6) Klingenberg RD, Homann N, Ludwig D. Type I intestinal lymphangiectasia treated successfully with slow-release octreotide. Dig Dis Sci 2003; 48: 1506-9. [Medline] [CrossRef]

7) Reichlin S. Somatostatin. N Engl J Med 1983; 309: 1495-501. [Medline] [CrossRef]

8) Hengl G, Prager J, Pointner H. The influence of somatostatin on the absorption of triglycerides in partially gastrectomized subjects. Acta Hepatogastroenterol (Stuttg) 1979; 26: 392-5. [Medline]

9) Sari S, Baris Z, Dalgic B. Primary intestinal lymphangiectasia in children: is octreotide an effective and safe option in the treatment? J Pediatr Gastroenterol Nutr 2010; 51: 454-7. [Medline] [CrossRef]

10) Kuroiwa G, Takayama T, Sato Y, et al. Primary intestinal lymphangiectasia successfully treated with octreotide. J Gastroenterol 2001; 36: 129-32. [Medline] [CrossRef] 